\begin{tabular}{|c|c|c|}
\hline \multicolumn{1}{|c}{${ }^{0} \mathrm{C}$} & $K$, gemessen & $K$, aus Gl. (2) \\
\hline 0 & - & 4,084 \\
20 & - & 3,634 \\
40 & - & 3,281 \\
80 & 2,794 & 2,771 \\
100 & 2,573 & 2,582 \\
120 & 2,422 & 2,422 \\
140 & 2,288 & 2,287 \\
160 & 2,173 & 2,171 \\
200 & 1,980 & 1,981 \\
300 & - & 1,668 \\
400 & - & 1,48 \\
\hline
\end{tabular}

Tab. 2. Gleichgewichtskonstanten der Reaktion $\mathrm{HDO}+\mathrm{H}_{2}=\mathrm{H}_{2} \mathrm{O}+\mathrm{HD}$.

ster Wert recht genau ein um etwa $15 \mathrm{cal} / \mathrm{Mol}$ höherer Betrag. Die genaue numerische Übereinstimmung mit der oben gemachten Angabe mag auf Zufall beruhen, der Wert für $\Delta E^{0}=918 \mathrm{cal} / \mathrm{Mol}$ wird wohl mit einer Genauigkeit von wenigen cal/Mol als gesichert gelten können. Dieser Wert stimmt mit dem von Th. F ö r s te r ${ }^{26}$ angegebenen in denkbar bester Weise überein ${ }^{27}$. Es ist zu erwarten, daß die empirisch gefundene und durch den Vergleich mit den theoretischen Überlegungen weiter belegte Beziehung

$$
K=0,7377 e^{928 / R T}
$$

die Werte der Gleichgewichtskonstante auch über das durch die experimentellen Befunde belegte Températurbereich hinaus etwa von $0^{\circ}$ bis $400^{\circ}$ mit einer Genauigkeit von rund $1 \%$ richtig wiedergibt. In der Tab. 2 sind die so berechneten Werte zusammen mit den Mittelwerten aus den Meßwerten (vgl. Tab. 1) angegeben.

In gleicher Weise kann aus einem Meßwert, z. B. für das Gleichgewicht $\mathrm{D}_{2} \mathrm{O}+\mathrm{H}_{2}=\mathrm{H}_{2} \mathrm{O}+\mathrm{D}_{2}$, die Differenz der Nullpunktsenergien berechnet werden. Der Meßwert bei $120^{\circ}$ ergibt, $K_{2}=K_{1}^{2}$ gesetzt,

$$
\Delta E^{0}=1819 \mathrm{cal} / \mathrm{Mol} \text {. }
$$

Aus den bei Förster bzw. bei Farkas für die einzelnen Molekel angegebenen Nullpunktsenergien folgt $\Delta E^{0}=1808 \mathrm{cal} / \mathrm{Mol}$. Die Übereinstimmung ist auch hier vollkommen.

\section{Nachtrag (Oktober 1948):}

Nach Fertigstellung der vorliegenden Arbeit sind im Euckenschen Institut (Göttingen) Messungen der Verteilungskonstanten bei höheren Temperaturen von $\mathrm{H}$. L e u ger ing ausgeführt worden, die die folgenden Werte ergaben:

$\begin{array}{rrrrrr}t: & 19 \tilde{j}^{0} & 385^{0} & 525^{0} & 80 \check{5}^{0} & 84 \check{a}^{\circ} \\ V: & 1,956 & 1,436 & 1,314 & 1,134 & 1,100\end{array}$

Abb.1, in die die Werte nachträglich eingezeichnet wurden, zeigt ihre vorzügliche Übereinstimmung mit den hier angegebenen Daten. Verf. ist sehr dankbar. für die Mitteilung dieser Werte vor ihrer Publikation.

${ }_{23}$ Th. F ö r ste r ${ }^{2}$.

${ }_{27}$ Der von A. Farkas in seinem Buch angegebene Wert (s. auch K. Clusius, Angew. Chem., N.F. 56, 241 [1943]) ist ein wenig niedriger. Der Unterschied im Wert der beiden Autoren rührt im wesentlichen von einer Verschiedenheit der angenommenen Nullpunktsenergie des $\mathrm{H}_{2} \mathrm{O}$-Moleküls her (13097 cal/Mol bei F a rkas, $13128 \mathrm{cal} /$ Mol bei F ör st e r). Nach einer frdl. privaten Mitt. von Hrn. H. Z e is e ergibt die genaueste Berechnung aus spektroskopischen Daten $\Delta E^{0}=926 \pm 60 \mathrm{cal} / \mathrm{Mol}$.

\title{
Der sterische Faktor bei der Rekombination von Methyl-Radikalen
}

\author{
Von R. A. Marcus und E.W. R. Steacie \\ Division of Chemistry, National Research Council, Ottawa, Canada \\ (Z. Naturforschg. 4a, 332—334 [1949]; eingegangen am 30. September 1948)
}

$\mathrm{I}_{\mathrm{au}}^{\mathrm{n}}$ einer früheren Veröffentlichung ${ }^{1}$ haben wir auf die Bedeutung der Kenntnis des sterischen Faktors bei elementaren Reaktionen von Kohlenwasserstoffen hingewiesen und die Behauptung aufgestellt, daß die gewöhnlichen Zwischenreaktionen, bei denen Atome und freie Radikale eine Rolle spielen, sehr viel niedrigere sterische Faktoren haben, als man früher annahm.

1 E. W. R. Steacie, B. de B. Darwent u. W. R. Trost, Faraday Soc. Discussion 1947, 2, 80.
Die Kenntnis des sterischen Faktors der Reaktion

$$
2 \mathrm{CH}_{3} \rightarrow \mathrm{C}_{2} \mathrm{H}_{6}
$$

ist auch von großer Bedeutung für die Feststellung des Reaktionsmechanismuts, aber das hierfür vorhandene Beweismaterial ist höchst widerspruchsvoll. Zwei Faktoren spielen eine Rolle. Der eine ist die wohlbekannte Dreierstoß-Bedingung der Rekombinations-Reaktionen. Diese dürfte streng gültig sein für die Rekombination von zwei 


\begin{tabular}{|c|c|c|c|c|c|}
\hline $\begin{array}{c}\text { Partialdruck } \\
\text { des } \\
\mathrm{Hg}\left(\mathrm{CH}_{3}\right)_{2} \\
\mathrm{~mm} \\
\end{array}$ & $\begin{array}{c}\text { Mittl. } \\
\text { Partialdruck } \\
\text { von NO } \\
\text { mm } \\
\end{array}$ & $\begin{array}{l}\text { Zeit } \\
\text { Min. }\end{array}$ & $\begin{array}{l}\frac{d}{d t}\left[\mathrm{C}_{2} \mathrm{H}_{6}\right] \\
\mathrm{ccm} / \text { Stde. }\end{array}$ & $\begin{array}{l}\frac{-d}{d t}[\mathrm{NO}] \\
\mathrm{ccm} / \text { Stde. }\end{array}$ & $\frac{\frac{-d}{d t}[\mathrm{NO}]}{[\mathrm{NO}]\left(\frac{d}{d t}\left[\mathrm{C}_{2} \mathrm{H}_{6}\right]\right)^{1 / 2}}$ \\
\hline $\begin{array}{l}40 \\
40 \\
40\end{array}$ & $\begin{array}{l}0,54 \\
0, \overline{41}\end{array}$ & $\begin{array}{r}98 \\
99 \\
130\end{array}$ & $\begin{array}{l}0,015 \\
0,36 \\
0,017\end{array}$ & $\begin{array}{l}0,99 \\
1,30\end{array}$ & $\frac{13}{16}$ \\
\hline
\end{tabular}

Tab. 1. Temperatur $25^{\circ} \mathrm{C}$.

Wasserstoffatomen, während sie von geringerer Bedeutung ist im Falle der Rekombination zweier komplexer Radikale, die viele innere Freiheitsgrade besitzen, um die überschüssige Energie aufzunehmen. In zweiter Linie können wir erwarten, daß echte sterische Effekte die Rekombinationsausbeute sehr komplexer Radikale herabsetzen. Es sind aber im Falle der verhältnismäßig einfachen Radikale Meinungsverschiedenheiten vorhanden, und die Schätzungen der Stoßausbeute der Reaktion

$$
2 \mathrm{CH}_{3} \rightarrow \mathrm{C}_{2} \mathrm{H}_{6}
$$

reichen von 0,1 bis $10^{-6}$ und darunter ${ }^{2}$.

Es schien daher wünschenswert, genauere experimentelle Abschätzungen zu erhalten. Dazu braucht man eine Reaktion von Methylradikalen, deren Stoßausbeute bekannt ist und deren Geschwindigkeit mit derjenigen von Reaktion (1) verglichen werden kann. Als geeignetste Reaktion erscheint

$$
\mathrm{CH}_{3}+\mathrm{NO} \rightarrow \mathrm{CH}_{3} \mathrm{NO} \text {. }
$$

Die Untersuchung stammt von $\mathrm{F}$ or s y th ${ }^{3}$ und ergab eine Stoßausbeute von $1,4^{-10^{-5}}$ bei $\mathrm{Zim}$ mertemperatur. Zwar ist die Genauigkeit dieser Schätzung nicht hoch, aber die Größenordnung scheint recht zuverlässig. Die vorliegende Arbeit gibt einen vorläufigen Bericht über einige Experimente über die Photolyse von Quecksilberdimethyl bei Anwesenheit von Stickoxyd, welche angestellt wurden, um die relativen Geschwindigkeiten der Reaktionen (1) bzw. (2) bei Zimmertemperatur festzustellen.

Bei Vorhandensein erheblicher Mengen von Stickoxyd wird die stationäre Konzentration der Methylradikale bei der Photolyse stark reduziert,

2 Eine Übersicht über früher erschienene Arbeiten findet sich in E. W. R. Steacie, Atomic and Free Radical Reactions', New York 1946.

3 J. S. A. F o r s'y th, Trans. Faraday Soc. 37, 312 [1941]. und die einzigen wichtigen Reaktionsstufen sind:

$$
\begin{aligned}
\mathrm{Hg}\left(\mathrm{CH}_{3}\right)_{2}+h v & \rightarrow \mathrm{Hg}+2 \mathrm{CH}_{3}, \\
2 \mathrm{CH}_{3} & \rightarrow \mathrm{C}_{2} \mathrm{H}_{6}, \\
\mathrm{CH}_{3}+\mathrm{NO} & \rightarrow \text { Endprodukte. }
\end{aligned}
$$

Hieraus ergibt sich für kleine Umsätze

$$
\frac{\frac{-d}{d t}[\mathrm{NO}]}{[\mathrm{NO}]\left[\frac{d}{d t}\left[\mathrm{C}_{2} \mathrm{H}_{6}\right]\right]^{1 / 2}}=\frac{k_{2}}{k_{1}^{1 / 2}} .
$$

Aus der Menge des gebildeten Athans und aus der Menge des verbrauchten Stickoxyds kann man so die relátiven Geschwindigkeiten der Reaktionen

$$
2 \mathrm{CH}_{3} \rightarrow \mathrm{C}_{2} \mathrm{H}_{6}
$$

und $\mathrm{CH}_{3}+\mathrm{NO} \rightarrow$ Endprodukte

bestimmen.

Experimentelle Bedingung ist die Bildung einer meßbaren Äthanmenge bei einer Konzentration des Stickoxyds, die niedrig genug ist, um den Verbrauch an Stickoxyd zu messen, jedoch nicht so niedrig, daß der örtliche Verbrauch durch die Reaktion zu stark wird. Diese Bedingungen sind bei Zimmertemperatur und Partialdrucken des Stickoxyds von der Größenordnung 0,05 bis $0,5 \mathrm{~mm}$ und Quecksilberdimethyl-Drucken von 5 bis $40 \mathrm{~mm}$ gegeben. Tab. 1 zeigt typische Ergebnisse bei $25^{\circ} \mathrm{C}$ mit ungefiltertem Licht aus einem Quecksilberbogen.

Man erhält also

$$
\begin{aligned}
\frac{k_{2}}{k_{1}^{1 / 2}} & =14 \mathrm{ccm}^{1 / 2} \text { Stde. } .^{-1 / 2} \mathrm{~mm}^{-1} \\
& =2,8 \cdot 10^{3} \mathrm{ccm}^{1 / 2} \mathrm{Mol}^{-1 / 2} \mathrm{sec}^{-1 / 2}
\end{aligned}
$$

für ein Reaktionsvolumen von $80 \mathrm{ccm}$.

Aus Forsyths Arbeit ergibt sich

$$
k_{2}=1,4 \cdot 10^{-5} \cdot 2 \cdot 10^{14} \mathrm{ccm} \mathrm{Mol}^{-1} \mathrm{sec}^{-1} \text {. }
$$


Wenn man die gleiche Stoßzahl für Reaktion(1) annimmt, so kann man setzen

$$
k_{1}=s_{1} \cdot 2 \cdot 10^{14} \mathrm{ccm} \mathrm{Mol}^{-1} \mathrm{sec}^{-1},
$$

wobei $s_{1}$ der sterische Faktor der Reaktion (1) ist.

Hieraus ergibt sich

$$
s_{1}=5 \cdot 10^{-3} .
$$

Aus verschiedenen Gründen ist dieser Wert ein Maximum:

a) Falls Spuren von Methan vorhanden sind, werden sie als Stickoxyd auftreten und den scheinbaren Verbrauch von NO verringern. Der Fehler aus dieser Quelle dürfte gering sein, da bei Vorhandensein erheblicher Mengen von NO die Methanbildung sehr klein ist.

b) Die verwandten Stickoxyd-Konzentrationen waren sehr klein, und es könnte trotz der Zirkulation der Gase das NO an einigen Stellen erschöpft gewesen sein. Dies würde für kurze Zeit eine zu starke Äthanbildung an den erschöpften Stellen zur Folge gehabt haben.

c) Es ist möglich, und es waren auch einige. Anzeichen dafür vorhanden, daß ein kleiner Bruchteil der Quecksilberdimethyl-Photolyse auch durch direkte molekulare Rückbildung Äthan ergibt nach den Gleichungen

$$
\begin{aligned}
\mathrm{Hg}\left(\mathrm{CH}_{3}\right)_{2}+h v & \rightarrow \mathrm{Hg}\left(\mathrm{CH}_{3}\right)_{2}{ }^{*} \\
& \rightarrow \mathrm{Hg}+\mathrm{C}_{2} \mathrm{H}_{6} .
\end{aligned}
$$

Eine solche Reaktion würde durch Stickoxyd nicht gehemmt. Wenn diese Reaktion in sehr geringem Ausmaße einträte, so würde sie den scheinbaren Ertrag von

relativ $\mathrm{zu}$

$$
2 \mathrm{CH}_{3} \rightarrow \mathrm{C}_{2} \mathrm{H}_{6}
$$

$$
\mathrm{CH}_{3}+\mathrm{NO} \rightarrow \text { Endprodukte }
$$

stark vergrößern.

Man kann daher wohl schließen, daß der sterische Faktor bei der Rekombination von Methylradikalen zur Bildung von Äthan gleich oder kleiner als $5 \cdot 10^{-3}$ ist. Wahrscheinlich ist er noch wesentlich kleiner, und man kann hoffen, daß die im Gang befindlichen Arbeiten noch endgültigere Ergebnisse liefern werden.

Man kann aus diesen Werten etwas über den sterischen Faktor der Reaktion

$$
\mathrm{CH}_{3}+\mathrm{H}_{2} \rightarrow \mathrm{CH}_{4}+\mathrm{H}
$$

entnehmen. Aus Experimenten über Photolyse von Quecksilberdimethyl bei Vorhandensein von $\mathrm{H}_{2}$ unter Bedingungen, die ähnlich wie die unsrigen waren ${ }^{4}$, erhält man

$$
\frac{k_{3}}{k_{1}^{1 / 2}}=2 \cdot 10^{-2} \mathrm{ccm}^{1 / 2} \mathrm{Mol}^{-1 / 2} \sec ^{-1 / 2},
$$

und daraus

$k_{3}=2 \cdot 10^{4} \mathrm{ccm} \mathrm{Mol}^{-1} \mathrm{sec}^{-1}$ bei Zimmertemperatur.

Wenn man $E_{3}=8,1 \mathrm{kcal}$ setzt, so ergibt sich $s_{3}=10^{-4}$ als Maximalwert, was mit den früheren Annahmen ${ }^{1}$ gut übereinstimmt.

Man kann den Schluß ziehen, daß im allgemeinen die sterischen Faktoren der meisten elementaren Reaktionen viel kleiner sind als die früher gewöhnlich angenommenen Werte 1 oder 0,1 . Obwohl keine speziellen Angaben vorliegen, möchten wir einen Wert von $10^{-4}$ bis $10^{-5}$ für die sterischen Faktoren einfacher freier Radikalreaktionen annehmen.

${ }_{4}^{4}$ M. K. Phibbs u. B. de B. Darwent, unveröff. Arbeit. 